\title{
Induction of DNA damage and apoptosis in human leukemia cells by efavirenz
}

\author{
ANSGAR BRÜNING $^{1 *}$, JULIA JÜCKSTOCK ${ }^{1 *}$, BERND KOST ${ }^{1}$, PANAGIOTIS TSIKOURAS ${ }^{2}$, \\ TOBIAS WEISSENBACHER ${ }^{1}$, SVEN MAHNER $^{1}$ and IOANNIS MYLONAS ${ }^{1}$ \\ ${ }^{1}$ Department of Gynecology and Obstetrics, Division of Infectious Diseases in Obstetrics and Gynecology,
Campus Innenstadt, Ludwig-Maximilians University of Munich, D-80337 Munich, Germany;
${ }^{2}$ Department of Obstetrics and Gynecology, Democrite University Thrace, 68100 Alexandroupolis, Greece
}

Received June 8, 2016; Accepted October 17, 2016

DOI: $10.3892 /$ or.2016.5243

\begin{abstract}
As part of the efforts to drug repurposing, some HIV drugs have recently been identified to exert anticancer effects. Selected nucleoside analogues of nucleosidic reversetranscriptase inhibitors (NRTIs) have been shown to interfere with RNA transcription of HI viruses as well as with the replication of DNA in cancer cells. Non-nucleosidic reverse transcriptase inhibitors (NNRTIs) are believed to have less effects on human DNA replication and, thus, on cancer cell proliferation. Assessment of the effect of the NNRTI efavirenz in human cancer cells, however, revealed a high sensitivity of leukemia cells to this agent at pharmacologically relevant concentrations of less than $10 \mu \mathrm{g} / \mathrm{ml}$. Cell death induced by efavirenz was caused by apoptosis, as shown by FACScan analysis (Annexin binding) and western blot analysis (cleavage of caspases and PARP). Western blot analyses also revealed a pronounced activation and phosphorylation of the DNA damage marker proteins p53, chk2 and H2AX, indicating DNA replication and genomic integrity as primary targets of efavirenz in leukemia cells.
\end{abstract}

\section{Introduction}

The increasing worldwide incidence of cancer is a serious global health problem. Despite significant advances in cancer treatment and drug development, there is an urgent need for additional and better tolerated anticancer drugs to treat primary and recurrent cancers. Unfortunately, the development and discovery of new anticancer drugs is expensive,

Correspondence to: Professor Ioannis Mylonas, Department of Gynecology and Obstetrics, Division of Infectious Diseases in Obstetrics and Gynecology, Campus Innenstadt, Ludwig-Maximilians University of Munich, Maistrasse 11, D-80337 Munich, Germany

E-mail: ioannis.mylonas@med.uni-muenchen.de

${ }^{*}$ Contributed equally

Key words: efavirenz, leukemia, apoptosis, DNA damage time-consuming, and in the worst case, must be terminated during clinical trials due to the occurrence of intolerable side effects or poor effectiveness $(1,2)$.

Testing of already approved therapeutic drugs for additional purposes, so-called drug repositioning, is a recently growing approach to discover new applications for already existing drugs $(1,3)$. When screening tests or preclinical data indicate a new possible application for a specific drug, preexisting knowledge on its pharmacokinetics, bioavailability, and side effects facilitate the assessment of its possible application in humans. Recent studies have indicated that HIV drugs may represent a valuable source of possible new anticancer drugs $(4,5)$. In particular, the HIV protease inhibitor nelfinavir was found to be highly active on a variety of human cancer cells (6-10), and is currently being tested in several clinical studies on cancer patients (7,11-13).

A screening of anti-retroviral drugs for their efficacy on human cancer cells revealed a striking sensitivity of cancer cells to the non-nucleoside reverse transcriptase inhibitor (NNRTI) efavirenz (14-17). Recently we observed that efavirenz could influence cell viability of endothelial cells (14). Moreover, this inhibition was associated with an increase in oxidative stress markers, endoplasmic reticulum (ER) stress markers, and autophagy (14). A cytotoxic effect has been demonstrated on several tumor cell lines including colorectal, pancreatic and the human leukemia cell lines Jurkat (acute T-cell leukemia cells) (17). Blood cancer cells are highly sensitive to cytostatic drugs but, depending on the cancer type, often become resistant after initial therapy, necessitating second and even third line treatment therapies $(7,11,18)$. Thus, there is a need for additional new anticancer drugs that induce specific cell death pathways in leukemia cells $(11,18)$. The present study describes the effect of efavirenz on the leukemia cancer cell lines Jurkat (acute T-cell leukemia), HL60 (acute promyelocytic leukemia), and IM9 (EBV-transformed B-lymphoblastoid).

\section{Materials and methods}

Cells and cell culture. The human leukemia cell lines IM9 (ATCC CCL-159), and HL60 (ATCC CCL-240) were purchased from ATCC/LGC Standards (Wesel, Germany). Jurkat cells were provided by U. Schleicher (Erlangen, Germany). Cells 
were cultured in RPMI-1640 medium supplemented with $10 \%$ fetal calf serum and antibiotics at $37^{\circ} \mathrm{C}$ in a humidified atmosphere with $5 \% \mathrm{CO}_{2}$ and was performed as previously described $(11,14)$. All cell culture reagents were from PAA Laboratories (Pasching, Austria).

Reagents. Efavirenz was from Bristol-Myers Squibb (Munich, Germany) and recovered from $50 \mathrm{mg}$ capsules by means of repeated ethanol extraction and speed-vac concentration (Eppendorf Concentrator 5301; Eppendorf, Hamburg, Germany). The efavirenz extract was finally dissolved in $1 \mathrm{ml}$ of ethanol to obtain a stock solution of $50 \mathrm{mg} / \mathrm{ml}$ in ethanol.

Chemo-sensitivity assays (ATP and MTT assay). To test viability of cancer cells, the bioluminescent ATP assay was performed as previously described (14). For the ATP assay, 5,000 cells in a total volume of $200 \mu \mathrm{l}$ were plated in flat bottom 96-well plates (Nunc, Wiesbaden, Germany) and incubated with the indicated cytostatic drugs for $48 \mathrm{~h}$ at $37^{\circ} \mathrm{C}$. For cell extraction, $50 \mu \mathrm{l}$ tumor cell extraction buffer (DCS Innovative Diagnostic Systems, Hamburg, Germany) was added to each well, mixed thoroughly and incubated for $20 \mathrm{~min}$ at room temperature. Leukemia cells were collected by centrifugation before extraction in $50 \mu \mathrm{l}$ cell extraction buffer. Using a MicroLumat LB 96P bioluminometer (EG\&G Berthold, Bad Wildbad, Germany), Luciferin-Luciferase agent (DCS Innovative Diagnostic Systems) was added automatically to each sample and analyzed for bioluminescence.

\section{FACScan analyses}

Annexin binding assay. FITC-labeled Annexin V (BioCat, Heidelberg, Germany) was applied to viable cells as recommended by the supplier in combination with propidium iodide and analyzed by FACScan with an FL-1 setting (propidium iodide) of $575 \mathrm{~nm}$ and an FL-2 setting (FITC) of $530 \mathrm{~nm}$. FACScan analysis was performed using a Beckman Coulter Epics XL-MCL flow cytometer (Beckman Coulter, Munich, Germany).

Cell cycle analysis. For cell cycle analysis, HL60 cells were treated for $24 \mathrm{~h}$ with efavirenz, washed with PBS, fixed with $70 \%$ methanol, and stained with $50 \mu \mathrm{g} / \mathrm{ml}$ propidium iodide in PBS, containing $1 \mathrm{mg} / \mathrm{ml}$ RNAse (Sigma, Munich, Germany) prior to FACScan analysis (620 nm filter).

Reactive oxygen species (ROS). Detection of ROS by FACScan analysis used 2',7'-dichlorodihydrofluorescein diacetate substrate (DCFH-DA; Sigma). HL60 cells were treated with efavirenz for $24 \mathrm{~h}$. Cells were then incubated for $45 \mathrm{~min}$ with $1 \mu \mathrm{g} / \mathrm{ml}$ of DCF-DA in cell culture medium under cell culture conditions, and subjected to FACScan analysis with a $575 \mathrm{~nm}$ filter for detection of green fluorescence.

Western blot analysis. For immunoblot analysis, cancer cells were cultured in $10 \mathrm{~cm}$ diameter cell culture plates, and cell extracts were prepared by cell lysis in RIPA-buffer $(50 \mathrm{mM}$ Tris, $\mathrm{pH}$ 8.0, $150 \mathrm{mM} \mathrm{NaCl}, 1 \% \mathrm{NP}-40,0.5 \%$ doxycholine, $0.1 \%$ SDS) as previously described $(11,14)$. Samples containing $20 \mu \mathrm{g}$ protein each as determined by the Bio-Rad Bradford assay (Bio-Rad, München, Germany) were subjected to SDS-polyacrylamide gel electrophoresis, and proteins were transferred to PVDF membranes in a Bio-Rad Mini Protean II

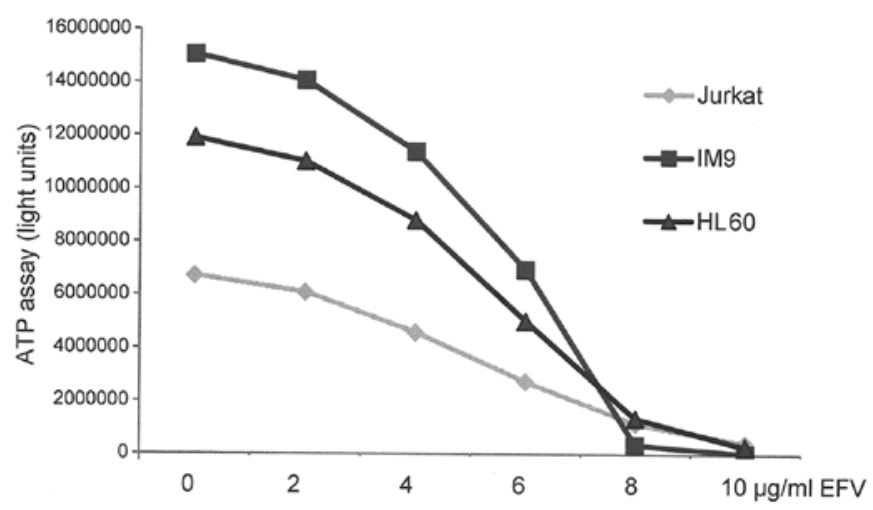

Figure 1. Efavirenz reduces cell viability of human leukemia cells. The leukemia cell lines HL60, IM9 and Jurkat were incubated with different concentrations of efavirenz for $48 \mathrm{~h}$ and analyzed for cell viability by the ATP assay.

(Bio-Rad) at $1 \mathrm{~mA} / \mathrm{cm}^{2}$ membrane in $10 \%$ methanol, $192 \mathrm{mM}$ glycine, $25 \mathrm{mM}$ Tris, pH 8.2. Membranes were blocked with $4 \%$ non-fat milk powder in PBS with $0.05 \%$ Tween-20 for $4 \mathrm{~h}$. Primary antibodies were applied in blocking buffer and incubated at room temperature overnight. All primary antibodies were purchased from Cell Signaling Technology (NEB, Frankfurt, Germany). Secondary, alkaline phosphatase (AP)-coupled antibodies against the corresponding primary antibodies were from Dianova (Hamburg, Germany). AP detection was performed by the chromogenic BCIP/NBT assay (Promega, Mannheim, Germany).

\section{Results}

Effect of efavirenz on cell survival and apoptosis in leukemiaderived cell lines. The three leukemia-derived cell lines HL60 (acute promyeolocytic leukemia),Jurkat (acute T-cell leukemia), and IM9 (B-lymphoblastoid) proved to be highly sensitive to efavirenz, revealing a near complete loss of cell viability at a concentration of $10 \mu \mathrm{g} / \mathrm{ml}$ of efavirenz (Fig. 1). Analysis of efavirenz-treated leukemia cells by microscopy revealed typical morphological signs of apoptosis, such as cellular fragmentation and membrane blebbing (Fig. 2). FACScan analysis of efavirenz-treated cancer cells by propidium iodide/Annexin staining confirmed the occurrence of apoptosis in addition to necrosis by biochemical means (Fig. 3).

Efavirenz induces activation of p53 and H2AX. To gain further insight into the molecular and cell biological effects of efavirenz in human cancer cells, western blot analysis of efavirenz-treated cancer cells was performed (Fig. 4). Increasing concentrations of efavirenz resulted in the cleavage of all relevant caspases of the intrinsic apoptotic pathway (Fig. 4), confirming the induction of the canonical apoptotic pathway. In IM9 and Jurkat cells, downregulation of the anti-apoptotic mitochondrial membrane protein mcl-1 was observed (Fig. 4). Enhanced phosphorylation of p53 and upregulation of the p53 target gene bak were observed in the p53 wild-type leukemia cell line IM9 (Fig. 4). As these effects were absent in the p53 negative cell lines Jurkat and HL60, further p53-independent pathways that contribute to cell death induced by efavirenz were investigated. Because phosphorylation of p53 in IM9 

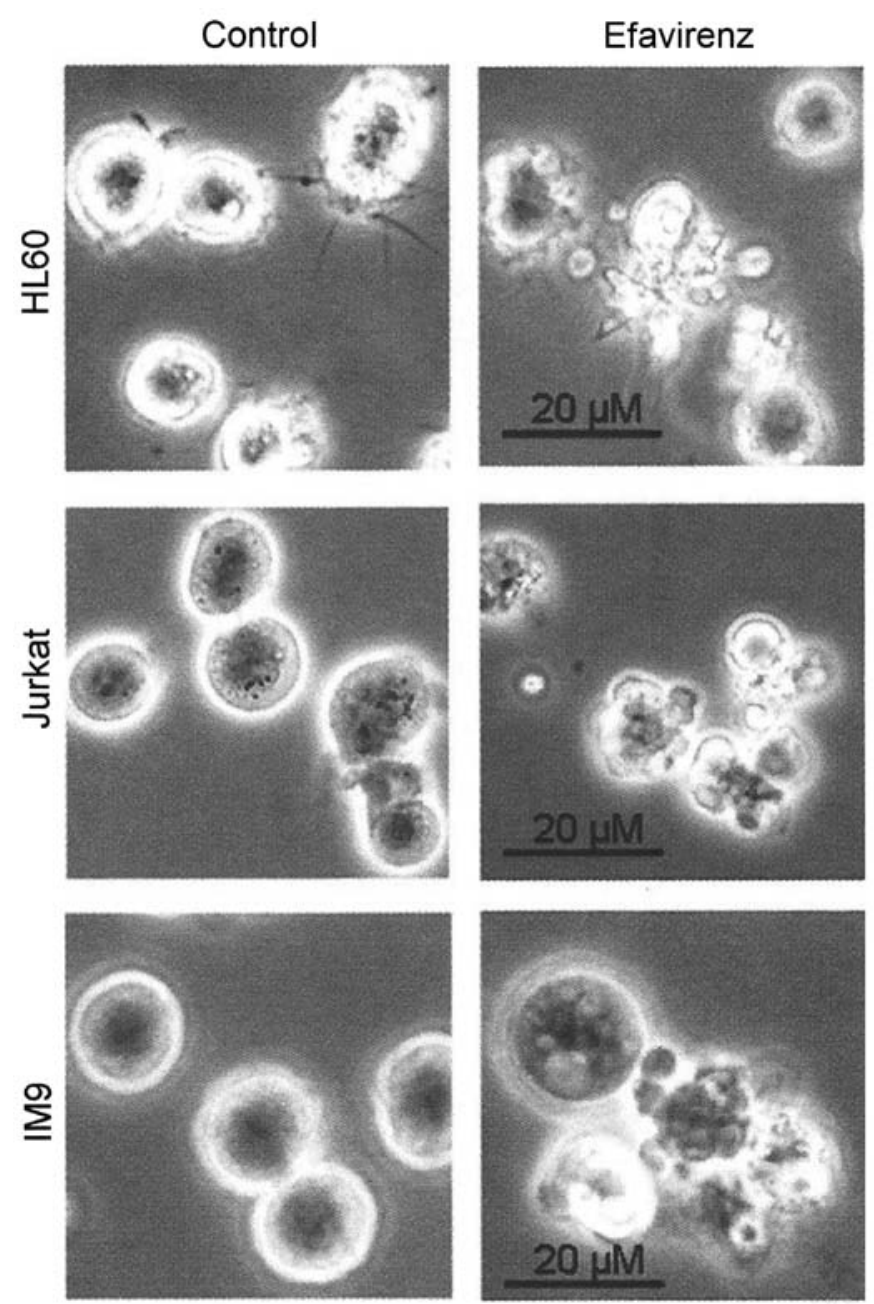

Figure 2. Efavirenz induces morphological signs of apoptosis in leukemia cells. The leukemia cell lines HL60, IM9 and Jurkat were treated with $8 \mu \mathrm{g} / \mathrm{ml}$ efavirenz and examined by phase contrast microscopy for the occurrence of morphological signs of apoptosis.

cells indicated induction of DNA damage, the expression and phosphorylation of the DNA damage-associated histone $\mathrm{H} 2 \mathrm{AX}$ in efavirenz-treated leukemia cells was tested. A strong increase in $\mathrm{H} 2 \mathrm{AX}$ phosphorylation $(\gamma \mathrm{H} 2 \mathrm{AX})$ was observed in efavirenz-treated Jurkat cells (Fig. 4) and, to a lesser extent, in IM9 and HL60 cells (Fig. 4).

Effect of efavirenz on cell cycle progression and generation of reactive oxygen species. Induction of DNA damage and activation of p53 often results in cell cycle arrest, although, in the presence of efavirenz, cell death by apoptosis appeared to prevail when higher concentrations of efavirenz were applied. In fact, the application of $5 \mu \mathrm{g} / \mathrm{ml}$ efavirenz increased the number of $\mathrm{G} 2 / \mathrm{M}$ phase cells from 14 to $20 \%$, whereas $10 \mu \mathrm{g} / \mathrm{ml}$ efavirenz predominantly caused cell death in HL60 cells, as visualized by a high percentage of sub-G1 phase cells, representing apoptotic or necrotic cells (Fig. 5). Since DNA damage may also be a direct, though unspecific effect of the generation of ROS, we also analyzed the generation of ROS by efavirenz. Unexpectedly, no generation of ROS could be detected in HL60 cells even when high concentrations $(10 \mu \mathrm{g} / \mathrm{ml})$ of efavirenz were applied (Fig. 5).
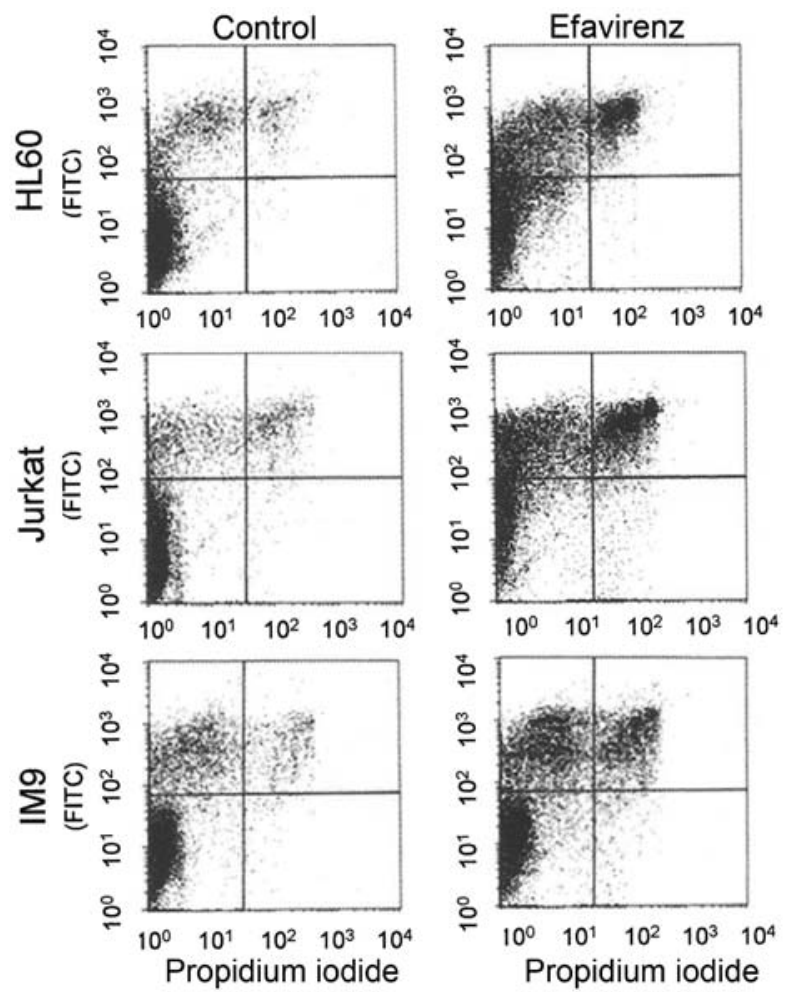

Figure 3. Efavirenz treatment causes apoptosis in leukemia cells. A FACScan analysis with propidium-iodide/FITC-Annexin stained leukemia cells was performed on the same cell lines (HL60, IM9 and Jurkat) for the detection of biochemical signs of apoptosis (FITC-Annexin staining) and necrosis (propidium-iodide staining).

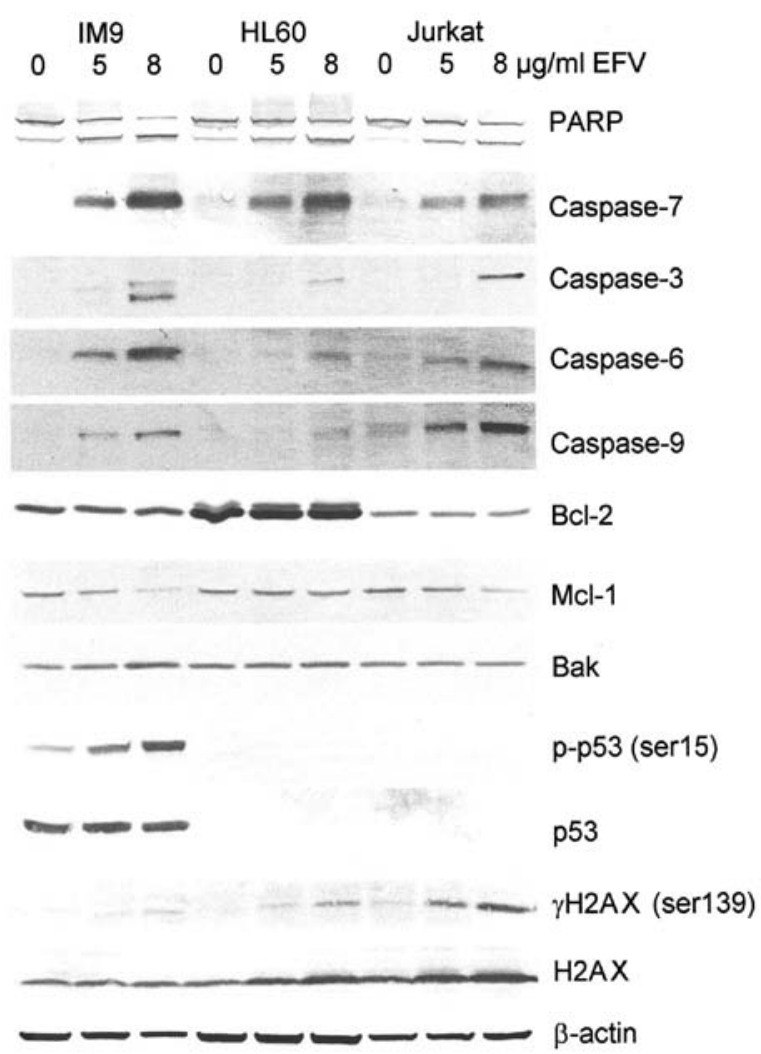

Figure 4. Efavirenz causes phosphorylation of p53 and H2AX. The leukemia cell lines IM9, HL60 and Jurkat were incubated for $24 \mathrm{~h}$ with either 5 or $8 \mu \mathrm{g} / \mathrm{ml}$ of efavirenz, and cell lysates were subjected to western blot analysis for the expression of apoptosis-relevant proteins. The cleaved versions of caspases are shown. 
A
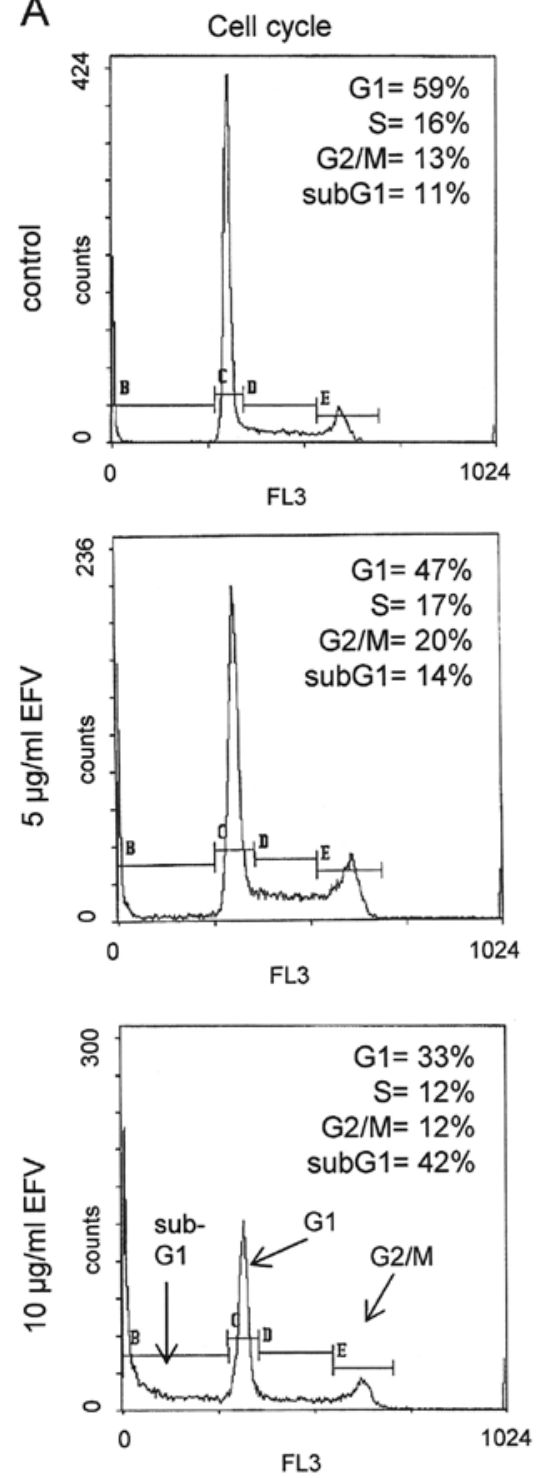

B
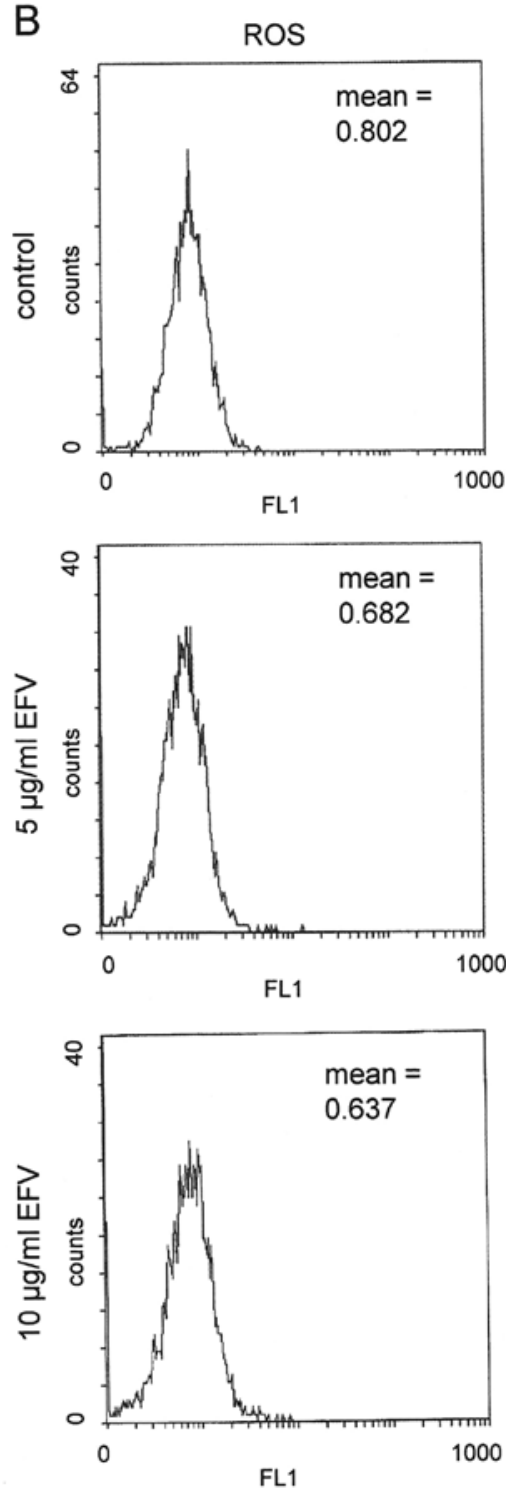

Figure 5. Effect of efavirenz on cell cycle progression and reactive oxygen species (ROS) generation. (A) HL60 cells were incubated for $24 \mathrm{~h} \mathrm{with} 5 \mathrm{or} 10 \mu \mathrm{g} / \mathrm{ml}$ of efavirenz, and analyzed for cell cycle distribution by FACScan analysis. The percentage of cells in the corresponding cell cycle phases are indicated within the figure. SubG1 phase cells: cell debris derived from apoptotic or necrotic cells. (B) HL60 cells were incubated for $5 \mathrm{~h}$ (when cell morphology was still intact) with 5 or $10 \mu \mathrm{g} / \mathrm{ml}$ of efavirenz, and generation of reactive oxygen species was detected by FACScan analysis using the fluorogenic substrate 2',7'-dichlorodihydrofluorescein diacetate. Mean fluorescence values are indicated within the figure.

\section{Discussion}

The results of the present study reveal that the HIV drug efavirenz effectively induces apoptosis in leukemia cells. This indicates a possible new perspective for the application of efavirenz in the treatment of human non-solid cancer. Efavirenz induces the classical apoptotic pathway associated with an enhanced phosphorylation of H2AX, suggesting efavirenz-induced DNA damage as a possible trigger for apoptosis. The H2AX histone subtype is not a DNA repair enzyme itself, but forms a platform and recognition site for DNA repair enzymes and DNA damage-associated signaling factors, especially at DNA double strand breaks $(19,20)$.

Recently we demonstrated that efavirenz can induce cell stress and reduced cell proliferation of endothelial cells (14). However, concentrations of up to $10 \mu \mathrm{g} / \mathrm{ml}$, which efficiently induce cell death in leukemia cells, left endothelial cells viable and did not destroy endothelial cell meshwork formations (14). Efavirenz has demonstrated a significant anti-proliferative effect in pancreatic cancer cells (15). A cytotoxic effect has been demonstrated on several tumor cell lines including colorectal, pancreatic and Jurkat leukemia cells (17). Interestingly, while a change in the phosphorylation of ERK and AKT was not observed, the tumor suppressor protein p53 revealed an increased activation of phosphorylation (17). Moreover, a synergistic effect with cannabinoid agonists has been observed (17).

The exact mechanism of efavirenz-induced DNA damage remains to be elucidated, especially since efavirenz does not represent a nucleoside analogue. The induction of oxidative stress has often been suggested as the mechanism mediating DNA damage caused by xenobiotics (21). Indeed, Apostolova et al recently described the induction of oxidative stress in efavirenz-treated human hepatocytes and hepatoma 
cells (22), and we have recently described enhanced oxidative stress by efavirenz in human endothelial cells (14). However, we did not observe the induction of oxidative stress in efavirenz-treated leukemia cancer cells as analyzed by the same standardized ROS assay. Therefore, the different tissue sensitivity against efavirenz resulting in oxidative stress still remains to be elucidated.

In a phase II trial with 53 patients with a metastatic castration-resistant prostate cancer (mCRPC), the use of an increased dosage regime of efavirenz may be beneficial for treatment (16). In the treatment of leukemia, efavirenz could be tested as a single agent, based on data showing that the effective drug concentrations $(\sim 10 \mu \mathrm{g} / \mathrm{ml})$ are not substantially higher than the doses currently used for the long-term treatment of HIV-infected persons receiving daily efavirenz treatment. The long-term pharmacological experience with this drug, its oral bioavailability, tolerable side effects, and its good compliance $(23,24)$ are further aspects that could facilitate the design and implementation of clinical trials on efavirenz with leukemia patients.

\section{Acknowledgements}

We would like to thank Mrs. Martina Rahmeh (Department of Obstetrics and Gynecology, Campus Grosshadern, LudwigMaximilians University of Munich) for excellent technical assistance.

\section{References}

1. Dueñas-González A, García-López P, Herrera LA, MedinaFranco JL, González-Fierro A and Candelaria M: The prince and the pauper. A tale of anticancer targeted agents. Mol Cancer 7: 82, 2008.

2. Zaenker KS and Entschladen F: Paving roads for new drugs in oncology. Recent Patents Anticancer Drug Discov 4: 137-145, 2009.

3. Gupta SC, Sung B, Prasad S, Webb LJ and Aggarwal BB: Cancer drug discovery by repurposing: Teaching new tricks to old dogs. Trends Pharmacol Sci 34: 508-517, 2013.

4. Bernstein WB and Dennis PA: Repositioning HIV protease inhibitors as cancer therapeutics. Curr Opin HIV AIDS 3 : 666-675, 2008

5. Chow WA, Jiang C and Guan M: Anti-HIV drugs for cancer therapeutics: Back to the future? Lancet Oncol 10: 61-71, 2009.

6. Gills JJ, Lopiccolo J, Tsurutani J, Shoemaker RH, Best CJ, Abu-Asab MS, Borojerdi J, Warfel NA, Gardner ER, Danish M, et al: Nelfinavir, A lead HIV protease inhibitor, is a broadspectrum, anticancer agent that induces endoplasmic reticulum stress, autophagy, and apoptosis in vitro and in vivo. Clin Cancer Res 13: 5183-5194, 2007.

7. Brüning A, Gingelmaier A, Friese K and Mylonas I: New prospects for nelfinavir in non-HIV-related diseases. Curr Mol Pharmacol 3: 91-97, 2010

8. Xiang T, Du L, Pham P, Zhu B and Jiang S: Nelfinavir, an HIV protease inhibitor, induces apoptosis and cell cycle arrest in human cervical cancer cells via the ROS-dependent mitochondrial pathway. Cancer Lett 364: 79-88, 2015.
9. Kushchayeva Y, Jensen K, Recupero A, Costello J, Patel A, Klubo-Gwiezdzinska J, Boyle L, Burman K and Vasko V: The HIV protease inhibitor nelfinavir down-regulates RET signaling and induces apoptosis in medullary thyroid cancer cells. J Clin Endocrinol Metab 99: E734-E745, 2014.

10. Sun L, Niu L, Zhu X, Hao J, Wang P and Wang H: Antitumour effects of a protease inhibitor, nelfinavir, in hepatocellular carcinoma cancer cells. J Chemother 24: 161-166, 2012.

11. Brüning A, Rahmeh M, Gingelmaier A and Friese K: The mitochondria-independent cytotoxic effect of nelfinavir on leukemia cells can be enhanced by sorafenib-mediated mcl-1 downregulation and mitochondrial membrane destabilization. Mol Cancer 9: 19, 2010.

12. Rengan R, Mick R, Pryma D, Rosen MA, Lin LL, Maity AM, Evans TL, Stevenson JP, Langer CJ, Kucharczuk J, et al: A phase I trial of the HIV protease inhibitor nelfinavir with concurrent chemoradiotherapy for unresectable stage IIIA/IIIB non-small cell lung cancer: A report of toxicities and clinical response. J Thorac Oncol 7: 709-715, 2012.

13. Shim JS, Rao R, Beebe K, Neckers L, Han I, Nahta R and Liu JO: Selective inhibition of HER2-positive breast cancer cells by the HIV protease inhibitor nelfinavir. J Natl Cancer Inst 104: 1576-1590, 2012

14. Weiss M, Kost B, Renner-Müller I, Wolf E, Mylonas I and Brüning A: Efavirenz causes oxidative stress, endoplasmic reticulum stress, and autophagy in endothelial cells. Cardiovasc Toxicol 16: 90-99, 2016.

15. Hecht M, Erber S, Harrer T, Klinker H, Roth T, Parsch H, Fiebig N, Fietkau R and Distel LV: Efavirenz has the highest anti-proliferative effect of non-nucleoside reverse transcriptase inhibitors against pancreatic cancer cells. PLoS One 10: $\mathrm{e} 0130277,2015$.

16. Houédé N, Pulido M, Mourey L, Joly F, Ferrero JM, Bellera C, Priou F, Lalet C, Laroche-Clary A, Raffin MC, et al: A phase II trial evaluating the efficacy and safety of efavirenz in metastatic castration-resistant prostate cancer. Oncologist 19: 1227-1228, 2014.

17. Hecht M, Harrer T, Büttner M, Schwegler M, Erber S, Fietkau R and Distel LV: Cytotoxic effect of efavirenz is selective against cancer cells and associated with the cannabinoid system. AIDS 27: 2031-2040, 2013.

18. Woyach JA and Johnson AJ: Targeted therapies in CLL: Mechanisms of resistance and strategies for management. Blood 126: 471-477, 2015.

19. Gochhait S, Dar S, Pal R, Gupta P and Bamezai RN: Expression of DNA damage response genes indicate progressive breast tumors. Cancer Lett 273: 305-311, 2009.

20. Ivashkevich A, Redon CE, Nakamura AJ, Martin RF and Martin OA: Use of the $\gamma-\mathrm{H} 2 \mathrm{AX}$ assay to monitor DNA damage and repair in translational cancer research. Cancer Lett 327: 123-133, 2012.

21. Monks TJ, Xie R, Tikoo K and Lau SS: Ros-induced histone modifications and their role in cell survival and cell death. Drug Metab Rev 38: 755-767, 2006.

22. Apostolova N, Gomez-Sucerquia LJ, Moran A, Alvarez A, Blas-Garcia A and Esplugues JV: Enhanced oxidative stress and increased mitochondrial mass during efavirenz-induced apoptosis in human hepatic cells. Br J Pharmacol 160: 2069-2084, 2010.

23. Best BM and Goicoechea M: Efavirenz - still first-line king? Expert Opin Drug Metab Toxicol 4: 965-972, 2008.

24. Maggiolo F: Efavirenz: A decade of clinical experience in the treatment of HIV. J Antimicrob Chemother 64: 910-928, 2009. 